\title{
Better defining the optimal management of penile urethral strictures: A retrospective comparison of single-stage vs. two-stage urethroplasty
}

Nathan Y. Hoy; David W. Chapman; Keith F. Rourke

Division of Urology, University of Alberta, Edmonton, AB, Canada

Cite as: Can Urol Assoc J 2019 April 26; Epub ahead of print. http://dx.doi.org/10.5489/cuaj.5895

Published online April 26, 2019

$* * *$

\section{Abstract}

Introduction: We aimed to compare single-stage and two-stage urethroplasty techniques for the treatment of penile urethral strictures.

Methods: We performed a retrospective review of all penile urethroplasties performed at a single centre between 2003 and 2017. The primary outcome was urethral patency, defined as the ability to easily pass a 16 Fr flexible cystoscope at six and 18 months of followup, and development of 90-day complications.

Results: Overall, 101 single-stage procedures (48 buccal mucosal graft [BMG] and 53 penile fasciocutaneous flap [PFF])and 53 two-stage procedures were performed. There was no difference in median stricture length between groups $(\mathrm{p}=0.25)$. Cox regression analysis did not identify stricture etiology, length, age, obesity, prior reconstruction, or urethroplasty technique to be associated with failure. Log-rank testing did not demonstrate a difference in success rates between surgical techniques (91\% [48/53] PFF vs. 83\% [40/48] BMG vs. 87\% two-stage [46/53]). Thirty-nine percent (60/154) of patients experienced a complication (51\% [27/53] PFF vs. 29\% [14/48] BMG vs. 36\% [19/53] two-stage). Multivariate analysis found urethroplasty technique to be the only factor associated with development of complication $(\mathrm{p}=0.02)$; odds ratio relative to BMG was 3.1 ( $p=0.009)$ for PFF and $1.4(p=0.43)$ for two-stage.

Conclusions: There appears to be little difference in success between penile urethroplasty techniques. The shift in technique to a single-stage BMG, when appropriate, appears to be founded on the basis of fewer operations for the patient, relative to a two-stage repair, and a lower complication profile, relative to single-stage PFF, without compromising success rates. 


\section{Introduction}

The optimal management of penile urethral strictures is a controversial topic for reconstructive urologists. At the heart of the debate lies two issues - whether a flap is superior to a graft and when a single-stage repair should be preferentially employed over a 2-stage repair.

Within the past few decades, there has been a surge in use of buccal mucosa grafts (BMG) for bulbar urethroplasties. ${ }^{1}$ Excellent long-term outcomes in the bulbar urethra have resulted in a subsequent paradigm shift in penile urethroplasty technique from pedicled penile fasciocutaneous flaps (PFF) to grafts, without substantial evidence to support this transition. Proponents of PFF argue that the pendulous urethra lacks the robust vascularity of the bulbar urethra to support a graft, while a PFF brings its own blood supply to compensate for this lack of vascularity. ${ }^{2}$ Contrastingly, grafts are much easier to harvest, with minimal donor site morbidity, faster to use, and the fears of poor graft take in the penile urethra are unfounded based on several reports. ${ }^{1,3,4}$

Traditionally, 2-stage repairs were utilized in complex cases involving lichen sclerosus, prior hypospadias repairs, and prior urethral reconstructions. However, this exposes patients to the morbidity of two procedures. ${ }^{5}$ Accordingly, there has been an expansion of indications for single stage repairs for more complex strictures in an attempt to minimize the discomfort and risks of a second procedure, plus the typical 6 months of waiting between stages.

The study objectives are to compare BMG and PFF techniques, and single-stage and 2stage urethroplasty for the repair of penile urethral strictures. We hypothesize that single stage urethroplasty with BMG offers similar success, with reduced morbidity, compared to staged and flap techniques. The approach to penile urethral strictures at our center has mirrored this wider paradigm shift, allowing us a unique opportunity to assess the impact this has had on urethroplasty outcomes.

\section{Methods}

We performed a retrospective review of penile urethroplasties performed at a single center between 2003-2017. Analysis included all patients who underwent penile urethroplasty. Ethics approval was obtained from our institutional review board. The primary outcome was urethral patency, defined as the ability to easily pass a 16Fr flexible cystoscope at 6 and 18 months follow-up. The secondary outcome was the development of 90-day complications during followup (classified by Clavien-Dindo system). For 2-stage procedures, complications for both the first and second stages were included.

\section{Surgical techniques}

Single-stage PFF

The penis is typically degloved via a circumcising incision. The urethra is spatulated ventrally beyond the proximal extent of the stricture. Stricture length is measured and an appropriately sized PFF is outlined. The flap margins are sharply incised through the dermis and the pedicle is 
mobilized from the Dartos fascia. The proximal anastomosis is performed and an onlay reconstruction is completed. A 16 French catheter is placed and the flap pedicle is spread fixed. The penile skin is closed and a dressing with occlusive gauze and gently compressive Coban ${ }^{\mathrm{TM}}$ wrap is applied. A voiding trial is performed 3 weeks post-operatively.

Single-stage BMG

A dorsal inlay or dorsal onlay technique is utilized depending on stricture location. A dorsal inlay is favoured for distal strictures while dorsal onlay is typically preferred for proximal strictures. The distal extent of the stricture is marked using a bougie-a-boule, followed by a semicircular subcoronal incision with dissection to Buck's fascia. An incision is made into the glans through the corpus spongiosum into the penile urethra. Distally, the urethra is spatulated to greater than 28 French. A stricturotomy is performed. The urethral plate is incised dorsally and spread fixed to the corpus cavernosum. A graft of appropriate dimensions is harvested. The BMG is spread fixed into the dorsal incision or dorsal corpus cavernosum. The corpus spongiosum is reconstructed and closed. Glans reconstruction, if necessary, after mobilizing glans wings, is completed. A 16 French catheter is placed. A dressing of non-adherent gauze, regular gauze, and a Coban ${ }^{\mathrm{TM}}$ wrapping is applied. A voiding trial is performed 3 weeks postoperatively.

\section{Two-stage BMG}

The first stage is performed similar to the one-stage BMG up to the stricturotomy step. At this point, a urethrostomy is created at the proximal portion of the stricturotomy. The urethral plate and the Dartos fascia and tunica albuginea alongside the plate are exposed. Bilateral BMGs are harvested based on the stricture length. These are secured along either side of the urethral plate. A 16 French catheter is placed. A bolster dressing composed of Xeroform ${ }^{\mathrm{TM}}$ gauze, surgical cotton soaked in mineral moisture, and regular gauze is then applied and secured with chromic sutures for 5 days. A trial of void is also performed on post-operative day 5 .

The second stage is undertaken six months later if there are no complications with graft maturation. A suprapubic catheter is placed under cystoscopic guidance. The urethral plate is outlined and the edges sharply incised. The urethral plate is closed over a 26 French sound. Glanuloplasty is completed with advancement of the glans flaps to the midline and the meatus is matured. A similar dressing to the one-stage PFF is then applied after a 14 French urethral stent is placed with a suprapubic catheter. A voiding trial is performed 3 weeks post-operatively.

\section{Statistical analysis}

Baseline patient and stricture characteristics were compared with Chi-square or Mann-Whitney testing where appropriate. Cox regression analysis was used to analyze factors associated with treatment failure. Kaplan-Meier regression modeling was used to demonstrate freedom from failure as a function of time. Binary logistic regression was then used to determine independent predictors of complications. Statistical tests were two-sided and a p-value $<0.05$ was considered 
significant. All analysis was performed on SPSS Software v24.0 (IBM Corp., Armonk, NY, USA).

\section{Results}

In total, 154 penile urethroplasties were included - 101 single-stage procedures [48 BMG and 53 PFF] and 53 2-stage procedures. Cohort demographics are outlined in Table 1. Lichen sclerosus was the most common etiology for BMG repairs (56\% [19/48]), compared to idiopathic for PFF repairs (26\% [14/53]), and hypospadias in the 2-stage repair (68\% [36/53]). There was no difference in median stricture length between groups at $5 \mathrm{~cm}$ (IQR 3.5-10) for PFF, $6.0 \mathrm{~cm}$ (3.6$9.8 \mathrm{~cm})$ for BMG, and $5.5 \mathrm{~cm}(4.0-7.0 \mathrm{~cm})$ for 2-stage $(\mathrm{p}=0.25)$ (Table 1).

Penile urethroplasty outcomes and complications are demonstrated in Table 2. Overall, success rates were comparable between the three groups at $91 \%(48 / 53)$ versus $83 \%(40 / 48)$, and 87\% (46/53) for PFF, BMG, and 2-stage techniques, respectively. Complications were experienced by 51\% [27/53] of the PFF group versus 29\% [14/48] for the BMG group versus $36 \%$ [19/53] of the 2-stage group. Within the PFF group, 17 complications were Clavien grade III and included 7 wound infections managed with antibiotics and 10 cases of ventral epidermolysis managed conservatively. Ten cases required re-intervention: one case of epidermolysis debrided under local anesthetic, 7 urethrocutaneous fistulas (UCF) surgically repaired, 1 ventral glans dehiscence requiring glanuloplasty, and 2 cases of flap necrosis debrided under a general anesthetic. In the BMG group, the 13 Clavien I-II complications included a urinary tract infection (UTI), pneumonia, hematoma, wound infection managed with antibiotics, 2 UCF, and 7 cases of epidermolysis managed conservatively. The single reoperative case was a combined UCF and ventral skin separation repair. The 12 Clavien I-II complications experienced in the 2-stage urethroplasty group, were 2 cases of epididymitis, hematuria requiring manual irrigation, urinary tract infection (UTI), occluded suprapubic catheter requiring exchange, painful BMG exposure, wound infection, 3 minor wound dehiscences, and 2 hematomas all managed conservatively. The complications requiring surgical intervention included a wound dehiscence, excision of symptomatic granulation tissue, and repair of $4 \mathrm{UCF}$.

Cox regression analysis did not find stricture etiology $(\mathrm{p}=0.76)$, length $(0.29)$, age $(p=0.24)$, obesity ( $p=0.07)$, prior reconstruction $(p=0.36)$, or urethroplasty technique $(p=0.35)$ to be associated with failure (Table 3). Kaplan-Meier plots and logrank testing did not demonstrate a difference in success rates between surgical techniques (91\% [48/53] PFF vs. 83\% [40/48] BMG vs. 87\% 2-stage [46/53]).

Binary logistic regression analysis found urethroplasty technique to be the only factor associated with development of complications ( $p=0.02)$. The OR for complications relative to BMG was 3.1 (95\% CI 1.33-7.30; p=0.009) for PFF and 1.4 (95\% CI 0.59-3.4; p=0.43) for 2stage urethroplasty (Table 4). 


\section{Discussion}

This retrospective cohort study represents the largest series comparing three different penile urethroplasty techniques. Available literature is fraught with limitations including inadequate follow-up, inaccurate specification of the stricture location (penile vs. bulbar), and heterogeneity in flap and graft origins. ${ }^{6}$ In our current study, success rates were similar across techniques, ranging from $83 \%$ for BMG to $91 \%$ for PFF.

\section{Buccal mucosal grafts vs. penile fasciocutaneous flaps}

There are 2 prospective trials randomizing patients to single stage BMG or PFF for anterior urethral strictures including both penile and bulbar stricture locations. ${ }^{7,8}$ No difference in success was found in these studies - 90\% BMG vs. 86\% PFF in Dubey et al., and 83\% BMG versus 90\% PFF in Soliman et al. Results from both trials are hampered by small sample sizes and heterogeneity between groups. If bulbar urethral strictures are excluded, the group sizes were only 19 for PFF and 20 for BMG in the first trial, and 11 for PFF and 13 for BMG in the second trial. The most common complications seen in these studies were post-void dribbling (PVD) in $15-32 \%$, superficial skin necrosis in $17-21 \%$, skin loss (6\%) and penile hypoesthesia in (11\%). ${ }^{7,8}$ PVD and penile hypoesthesia were not reported in our series, while focal flap necrosis rate was $4 \%$ and the epidermolysis rate was comparable at $21 \%$. The most common BMG complications in the Dubey trial were oral in $26 \%$, including increased salivation and perioral numbness. ${ }^{7}$ In the Soliman series, oral complications were seen in $32 \% .{ }^{8}$ In this present study, there were no reported oral complications, though a complication such as perioral numbness would depend on how patients were counselled regarding the donor site and length of follow-up. We did not consider it a complication if it resolved within 90 days. These series, and our data, highlight the advantages of BMG and shortcomings of PFF. The PFF is a complex flap with long suture lines which predispose to urethrocutaneous fistula (UCF) (13\%), epidermolysis (32\%), and rarely, dehiscence (2\%) and necrosis (4\%). Oral BMG complications are generally transient and our rates of UCF and epidermolysis were lower than with PFF (4\% and 15\%, respectively). Technically, harvesting and performing a BMG is easier than a PFF. On balance, it appears that single-stage BMG techniques offer similar success rates to PFF procedures, but incur lesser morbidity.

\section{Single-stage vs. staged penile urethroplasty}

In the largest series comparing single-stage and 2-stage techniques, Andrich et al. reported their experience with 1032 -stage repairs ( $\mathrm{N}=92$ after pure bulbar strictures excluded) and 139 singlestage repairs (20 PFF). ${ }^{5}$ A 2-stage technique was employed for lichen sclerosus, after previous failed hypospadias surgery, or when the urethral lumen was obliterated. The 6-month recurrence rate was $3 \%$ with single-stage, compared to $4 \%$ with 2-stages. Explanations for these lower failure rates include a shorter length of follow-up, inclusion of a significant number of bulbar strictures in both groups, and strict utilization of a 2-stage repair for all hypospadias revisions and lichen sclerosus. Another notable difference is the revision rate of the Andrich series. It was 
highest for 2-stage repairs at $38 \%$ and $20 \%$ for PFF. ${ }^{5}$ Though our PFF revision rate was similar (19\%), the main indications in our series were for UCF repairs and focal flap necrosis, whereas all the PFF revisions were for hematomas in the comparative study. Contrastingly, our 2-stage repair revision rate was only $11 \%$. The most common indication for revision in the referenced study was urethrostomy stenosis (18\%) and deepening of the glans cleft (13\%). ${ }^{5}$ The most common indication for reoperation in this series was for UCF after the second stage. The authors postulate the high revision rate may be secondary to erections in the early postoperative period leading to reactive hemorrhage and causing tension on the skin edges. It is our practice to leave patients on diazepam $5 \mathrm{mg}$ orally three times daily for 48 hours postoperatively, then discharge them on $5 \mathrm{mg}$ nightly for 3 weeks. Though it is unlikely this would account for the entirety of the discrepancy, it may lead to a lower revision rate. The main reason we have moved away from stringently using 2-stage repairs is 2-fold. Firstly, all patients are subjected to at least 2 procedures and for some, left with a cosmetically unsatisfactory penis for 6 months while awaiting the second stage. Secondly, approximately $11-50 \%$ will receive three or more operations as a result of complications requiring revisions. ${ }^{5}$ It appears the move from two-stage urethroplasties has been validated by similar outcomes, while reducing the total number of surgeries required.

\section{Study strengths}

A strength of our study is the utilization of Cox regression analysis to determine factors associated with treatment failure. Studies of penile strictures generally have a relatively heterogenous population with respect to stricture length, etiology, and prior surgeries. We were able to demonstrate that, despite the heterogeneity in these variables, none proved to be associated with treatment failure. Likewise, binary logistic regression confirmed urethroplasty technique, namely PFF, to be the only factor predictive of complications. Another strength of this study is the robust follow-up. As the only reconstructive center in a large catchment area, we had a relatively captive cohort and were able to document long term follow-up ranging from 2.5 years for BMG to 7 years for the PFF and 2-stage groups. Overall, this represents one of the largest series of patients with penile urethral strictures and avoids the inclusion of any pure bulbar strictures.

\section{Study limitations}

Though our practice has shifted towards utilizing a single-stage BMG preferentially to a PFF and 2-stage repair, there is still significant selection bias. A 2-stage repair remains our treatment of choice for failed single-stage repairs, an obliterated urethral plate, and for cases with a poorly developed glans. The challenge in generalizing these decision criteria are that the latter two are subjective surgeon assessments. There was baseline heterogeneity in stricture etiology between groups. However, on our regression analysis, stricture etiology was not associated with failure. A prospective randomized trial comparing these 3 techniques will likely never be feasible due to this being a relatively uncommon condition, resulting in small numbers necessitating the 
combination of varying stricture etiologies, and inherent surgeon biases in selecting 2-stage repairs for those strictures they deem to be more complex.

\section{Conclusion}

There appears to be little difference in success for penile urethroplasty between single-stage BMG, PFF, and 2-stage urethroplasties. Complication rates were highest with single-stage PFF. The shift in technique to a single-stage BMG, when appropriate, appears to be founded on the basis of less operations for the patient, relative to a 2-stage repair, and a lower complication profile, relative to single-stage PFF, without compromising success rates. 


\section{References}

1. Bhargava S, Chapple CR. Buccal mucosal urethroplasty: Is it the new gold standard? BJU Int 2004; 93:1191-3.

2. Carney KJ, McAninch JW. Penile circular fasciocutaneous flaps to reconstruct complex anterior urethral strictures. Urol Clin North Am 2002;29:397-409.

3. Marshall SD, Raup VT, Brandes SB. Dorsal inlay buccal mucosal graft (Asopa) urethroplasty for anterior urethral stricture. Transl Androl Urol 2015;4:10-5.

4. Asopa HS, Garg M, Singhal GG, et al. Dorsal free graft urethroplasty for urethral stricture by ventral sagittal approach. Urology 2001;58:657-9.

5. Andrich DE, Greenwell TJ, Mundy AR. The problems of penile urethroplasty with particular reference to 2-stage reconstructions. J Urol 2003;170:87-9.

6. Barbagli G, Lazzeri M. Surgical treatment of anterior urethral stricture diseases: brief overview. Int Braz J Urol 2007;33:461-9.

7. Dubey D, Vijjan V, Kapoor R, et al. Dorsal onlay buccal mucosa versus penile skin flap urethroplasty for anterior urethral strictures: results from a randomized prospective trial. $J$ Urol 2007;178:2466-9.

8. Soliman MG, Abo Farha M, El Abd AS, et al. Dorsal onlay urethroplasty using buccal mucosa graft versus penile skin flap for management of long anterior urethral strictures: a prospective randomized study. Scand J Urol 2014;48:466-73.

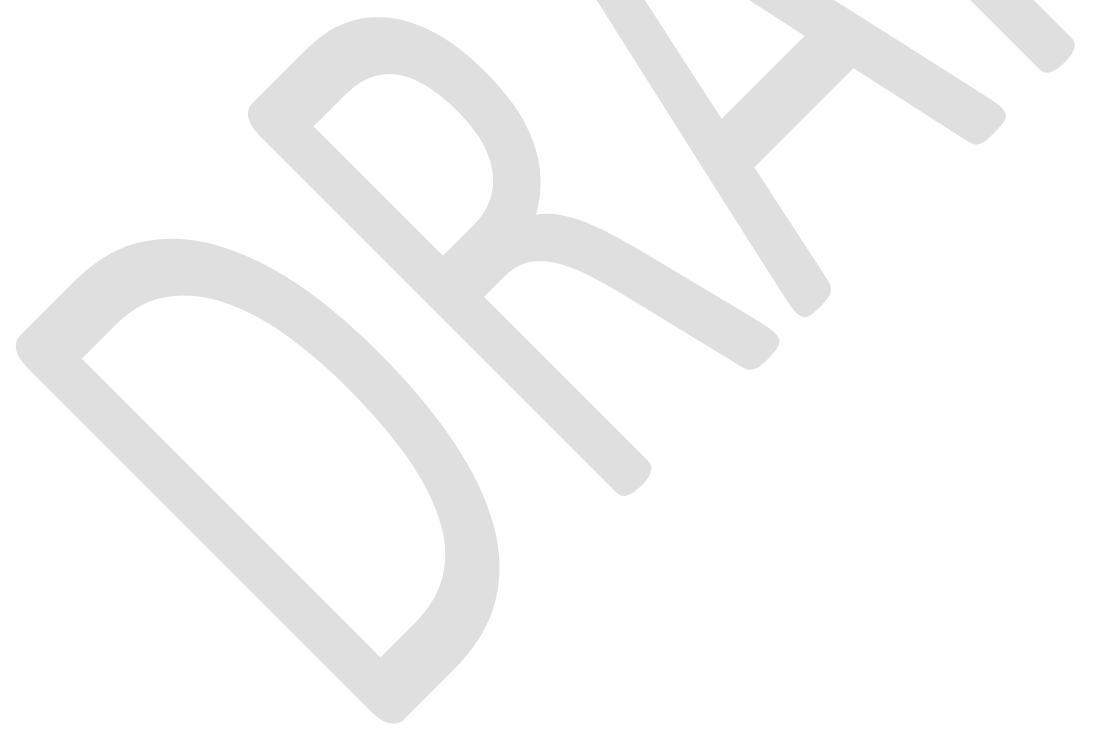


Figures and Tables

\begin{tabular}{|c|c|c|c|c|c|}
\hline \multicolumn{6}{|c|}{ Table 1. Baseline clinical characteristics } \\
\hline Clinical characteristic & $\begin{array}{c}\text { 1-stage } \\
\text { PFF, } \\
\text { n } \\
(\%, \text { IQR) }\end{array}$ & $\begin{array}{c}\text { 1-stage } \\
\text { BMG, } \\
\text { n (\%, IQR) }\end{array}$ & $\begin{array}{c}\text { 1-stage } \\
\text { overall, } \\
\text { n }(\%, \text { IQR) }\end{array}$ & $\begin{array}{c}\text { 2-stage, } \\
\text { n (\%, IQR) }\end{array}$ & $\mathbf{p}$ \\
\hline $\mathrm{n}$ & 53 & 48 & 101 & 53 & - \\
\hline $\begin{array}{l}\text { Mean age at urethroplasty } \\
\text { (years) }\end{array}$ & 49 & 50 & 49 & 35 & 0.0001 \\
\hline Median stricture length (cm) & $\begin{array}{c}5.0 \\
(3.5-10) \\
\end{array}$ & $\begin{array}{c}6.0 \\
(3.6-9.8) \\
\end{array}$ & $\begin{array}{c}5.0 \\
(3.5-10.0) \\
\end{array}$ & $\begin{array}{c}5.5 \\
(4.0-7.0) \\
\end{array}$ & 0.25 \\
\hline \multicolumn{6}{|l|}{ Stricture etiology } \\
\hline Idiopathic & $14(26.4)$ & $8(16.7)$ & $22(21.8)$ & $1(1.9)$ & $0.001^{*}$ \\
\hline Traumatic & $7(13.2)$ & $4(8.3)$ & $11(10.9)$ & $1(1.9)$ & $0.048^{*}$ \\
\hline Lichen sclerosus & $1(1.9)$ & $19(39.6)$ & $20(19.8)$ & $14(26.4)$ & 0.35 \\
\hline Radiation & $1(1.9)$ & $1(2.1)$ & $2(2.0)$ & $0(0)$ & 0.30 \\
\hline Hypospadias & $13(24.5)$ & $7(14.6)$ & $20(19.8)$ & $36(67.9)$ & $0.0001^{*}$ \\
\hline Iatrogenic & $16(30.2)$ & $9(18.8)$ & $25(24.8)$ & $1(1.9)$ & $0.0003^{*}$ \\
\hline Infectious/inflammatory & $1(1.9)$ & $0(0)$ & $1(0.99)$ & $0(0)$ & 0.47 \\
\hline Prior urethral reconstruction & $8(15.1)$ & $9(18.8)$ & $17(16.8)$ & $35(66.0)$ & $0.0001^{*}$ \\
\hline $\begin{array}{l}\text { Median length of followup } \\
\text { (months) }\end{array}$ & $\begin{array}{c}93 \\
(60-113) \\
\end{array}$ & $\begin{array}{c}32 \\
(18-50) \\
\end{array}$ & $\begin{array}{c}56 \\
(28-103) \\
\end{array}$ & $\begin{array}{c}87 \\
(45-121) \\
\end{array}$ & \\
\hline
\end{tabular}

p $<0.05$ denotes statistical significance. BMG: buccal mucosal graft; IQR: interquartile range; PFF: penile fasciocutaneous flap. 


\begin{tabular}{|c|c|c|c|c|c|}
\hline Outcome & $\begin{array}{c}\text { 1-stage } \\
\text { PFF, } \\
\text { n (\%) }\end{array}$ & $\begin{array}{l}\text { 1-stage } \\
\text { BMG, } \\
\text { n (\%) }\end{array}$ & $\begin{array}{c}\text { 1-stage } \\
\text { overall, } \\
\text { n (\%) }\end{array}$ & $\begin{array}{c}\text { 2-stage, } \\
\text { n (\%) }\end{array}$ & $\mathbf{p}$ \\
\hline $\begin{array}{l}\text { Successful } \\
\text { urethroplasty }\end{array}$ & $48(90.6)$ & 40 (83.3) & $88(87.1)$ & 46 (86.8) & 0.95 \\
\hline $\begin{array}{l}\text { Complication } \\
\text { experienced }\end{array}$ & $27(50.9)$ & $14(29.2)$ & $41(40.5)$ & 19 (35.8) & 0.57 \\
\hline \multicolumn{6}{|l|}{ Clavien grade } \\
\hline $\mathrm{I}$ & 10 (18.9) & $9(18.8)$ & $19(18.9)$ & $7(13.2)$ & 0.38 \\
\hline II & $7(13.2)$ & $4(8.3)$ & $11(10.9)$ & $5(9.4)$ & 0.79 \\
\hline IIIa & $1(1.9)$ & $0(0)$ & $1(1.0)$ & $0(0)$ & 0.47 \\
\hline IIIb & $9(17.0)$ & $1(2.1)$ & $10(9.9)$ & $6(11.3)$ & 0.93 \\
\hline IVa & $0(0)$ & $0(0)$ & $0(0)$ & $0(0)$ & - \\
\hline IVb & $0(0)$ & $0(0)$ & $0(0)$ & $0(0)$ & - \\
\hline $\mathrm{V}$ & $0(0)$ & $0(0)$ & $0(0)$ & $0(0)$ & - \\
\hline $\begin{array}{l}\text { Re-operation for } \\
\text { complication }\end{array}$ & $10(18.9)$ & $1(2.1)$ & $11(10.9)$ & $6(11.3)$ & 0.94 \\
\hline
\end{tabular}

BMG: buccal mucosal graft; PFF: penile fasciocutaneous flap.

\begin{tabular}{|l|c|c|c|}
\hline \multicolumn{4}{|c|}{ Table 3. Cox regression analysis of variables associated with treatment failure } \\
\hline Clinical variable & Hazard ratio & $\mathbf{9 5 \%}$ CI & p \\
\hline Age (years) & 0.99 & $0.95-1.01$ & 0.24 \\
\hline Obesity (BMI $\left.\geq 35 \mathrm{~kg} / \mathrm{m}^{2}\right)$ & 2.6 & $0.94-7.1$ & 0.07 \\
\hline Stricture length $(\mathrm{cm})$ & 1.1 & $0.94-1.24$ & 0.29 \\
\hline Previous urethroplasty & 0.62 & $0.23-1.72$ & 0.36 \\
\hline Technique & & & 0.36 \\
\hline 1-stage BMG & Referent & - & - \\
\hline 1-stage PFF & 0.44 & $0.14-1.4$ & 0.15 \\
\hline 2-stage & 0.64 & $0.23-1.8$ & 0.39 \\
\hline Stricture etiology & & & 0.98 \\
\hline Idiopathic & Referent & - & - \\
\hline Traumatic & 1.6 & $0.22-11.0$ & 0.66 \\
\hline Lichen sclerosus & 1.9 & $0.39-9.6$ & 0.42 \\
\hline Radiation & 1.0 & $0.9-1.01$ & 0.99 \\
\hline Hypospadias & 1.3 & $0.26-6.1$ & 0.77 \\
\hline Iatrogenic & 1.1 & $0.19-6.8$ & 0.89 \\
\hline Inflammatory/infectious & 1.0 & $0.99-1.01$ & 0.99 \\
\hline
\end{tabular}

BMG: buccal mucosal graft; BMI: body mass index; CI: confidence interval; PFF: penile fasciocutaneous flap. 


\begin{tabular}{|c|c|c|c|}
\hline Clinical variable & Odds ratio & $95 \% \mathrm{CI}$ & $\mathbf{p}$ \\
\hline Age & 0.98 & $0.96-1.00$ & 0.07 \\
\hline Stricture length & 1.00 & $0.89-1.11$ & 0.93 \\
\hline Etiology & 0.95 & $0.79-1.15$ & 0.63 \\
\hline Obesity $\left(\mathrm{BMI} \geq 35 \mathrm{~kg} / \mathrm{m}^{2}\right)$ & 0.60 & $0.20-1.73$ & 0.34 \\
\hline Previous urethroplasty & 0.69 & $0.34-1.40$ & 0.30 \\
\hline Technique & & & $0.02^{*}$ \\
\hline 1-Stage BMG & Referent & - & - \\
\hline 1-Stage PFF & 3.1 & $1.33-7.30$ & $0.009^{*}$ \\
\hline 2-Stage & 1.4 & $0.59-3.40$ & 0.43 \\
\hline
\end{tabular}

$\mathrm{p}<0.05$ denotes statistical significance. BMG: buccal mucosal graft; BMI: body mass index; CI: confidence interval; PFF: penile fasciocutaneous flap. 